\title{
Artistic and Cultural Significance of Ghanaian Printed Fabric Designs
}

\author{
Emmanuel RK Amissah ${ }^{1 *}$ and Sylvia Mawunyo Letcher-Teye ${ }^{2}$ \\ ${ }^{1}$ Department of Textile Design and Fashion Studies Education, University of Education, Ghana
}

${ }^{2}$ Accra Girls' Senior High School, Private Mail Bag, Achimota, Ghana

Submission: December 17, 2017; Published: February 05, 2018

*Corresponding author: Emmanuel RK Amissah, Department of Textile Design and Fashion Studies Education, University of Education, Winneba, PO.Box 25, Winneba, Ghana, Email: erk.amissah@gmail.com

\begin{abstract}
The main objective of the research is to examine the artistic and cultural significance of Ghanaian printed fabrics. The study specifically sought to identify the various Ghanaian printed fabrics within the Ghanaian cultural set-up, examine the artistic and cultural significance of the fabrics and appraise some aspects of the printed fabrics. The research design used was the qualitative approach based on a simple descriptive survey. The purposive sampling technique was used to select eight (8) fabric designs for the study. Interview and observation were used in collecting data. The analysis of the study was based on a pictorial view of the designs. This technique enabled the researchers to deal with the artistic study of the research. The findings of the study showed that there are many Ghanaian printed fabrics with different names that have cultural significance derived from various traditional values and beliefs. Artistically, the fabrics come in different colour-ways, providing a venue for a variety of aesthetic experience for their users. The simple and complex designs associated with these fabrics go a long way to provide the individual with numerous means of appreciating textile products. The main recommendation of the study is that, conscious efforts be made by the various centres of national cultures to have special collections of these fabrics as a means of beefing up the stock of knowledge that exists in the meanings of the fabric designs.
\end{abstract}

Keywords: Artistic; Culture; Aesthetics; Fabrics; Colour-ways; Symbolism

\section{Introduction}

The design of fabrics is one of the essential commodities that need critical thinking and purposive approach in terms of their production. In Ghana, types of cloths and the design of fabrics are more than fashion. Fabrics represent status and symbolises many things depending on particular cultures. Ghanaian printed fabrics are worn mainly for funerals, durbars, naming ceremonies and other important celebrations. The distinctive, simple and intricate designs with a variety of colour-ways give something truly extraordinary with a rich cultural meaning. Ghana has one of the richest and most vibrant cultures in the world. This is embedded in the way of life of the people. Significant in this is shown in the many and various printed fabrics used by the people. These fabrics that come in various colour-ways with both simple and complex designs reflect the socio-cultural and artistic ways of its users. This is very significant in the Ghanaian culture as they are mostly used on specific occasions as well as everyday wear throughout the year. The Ghanaian printed fabrics have taken a global trend.

The fabrics are seen in almost all the continents where Ghanaians are present. They are used to portray the rich culture of the Ghanaian. These fabric designs possess special qualities of aesthetics that blend beautifully the nature of art, beauty and taste within the environment. It is among these qualities that cultural significance is embedded. Principles of design are adhered to in the designs of these printed fabrics. Tucker [1] indicated that the principle of design (that is, the unique arrangement of the element) is based on some aesthetic system. The principles of design govern the relationships of the elements used and organize the composition as a whole. Whether working with existing furnishings and fabrics or "starting from scratch" with an empty room, the designer should always use the elements and principles of design as a guide in choosing everything. The designer uses elements as tools or raw materials to design, much like paints are the basics to a painter. The elements of design include space, line, form, colour, dot, shape and texture. The principles of design relate to how these elements are utilised.

The principles of design are essential to the development and production of clothing used by individuals and families around the world. Each principle has a specific role in creating an aesthetically pleasing fabric or prints. The principles of design consist of balance, proportion (also referred to as scale), emphasis, rhythm and harmony. When a designer uses the elements and principles of design to create a visual unity, harmony is achieved. The designs embedded in these fabrics are often integrated in more than one principle bringing out 
the uniqueness of such fabrics. The use of fabrics in Ghana is a lifestyle, the essence of culture. Hofstede [2] was of the opinion that, culture is the cultivated behaviour that is socially transmitted. It involves the accumulation of knowledge, beliefs, attitudes, experiences, religion, relations, concepts of the universe, values, meanings, roles, notions of time, relations, material possessions and objects acquired and a way of life of a group of people throughout the generations.

This way of life is accepted by all the individuals in the group without thinking about it and is passed from one generation to another through communication and imitation. Culture is a lifestyle and it depicts the lifestyle of a group of people. By implication, it is man-made, not genetically inherited. It therefore originates as human response to the local physical and biological environment. The use of printed fabrics in Ghana has a peculiar history that has been handed down to present generation and generations to come. Culture is also manifested in tangible or material form, in terms of types of food procurement and preparation, diverse technology and crafts, clothing, body decoration, visual art and symbols as well as secular and religious architecture. Symbols play a great significance in the fabric design process. They give meanings to the designs created as expressed in the cultural settings of the people. Every symbol stands for and expresses a specific meaning or special value given to it. A common way of showing something as a symbol is a matter of sensory data into meaningful mental images. For example the lion by its nature is ferocious, fearful, strong animal, whose resentment would be dangerous if aroused. It represents a symbol of majesty, strength, military might and deathless courage. When referred to as 'the king of the beast' it stands for kingly power and might. However a symbol being referred to as a sign here may not be most appropriate. A symbol generally stands for some abstract idea.

What is expressed in symbols must be thought to be worth saying. What is symbolized is something of value. Symbols have turned out to be interwoven with history and life. Symbols help one to refer to an event that has long happened, and permit us to think about something, even if it is not presently available. Making of symbols are purely an art work, whether produced by artist or non-artist. It involves creativity in transforming emotions and concepts into art forms, so that all the qualities we see and perceive in terms of beauty can be made manifest. Any artwork, for that matter, a symbol, must be aesthetically appealing, meaningful and have a reason for being created. These have been the basis for ideas of motifs in the designs of Ghanaian printed fabrics. Colour symbolism also plays a very critical role in the textile design industry. Textile design cannot be complete without taking the meanings that the various colours express in the designs. It is therefore imperative that meanings of colour are dealt with to justify their uses in various designs. According to Amenuke SK, et al. [3] "Indigenous meanings assigned to colour are not based on modern scientific theories but on philosophical, psychological, and spiritual meanings related to life." This assertion indicates that colour in the Ghanaian context, is used to express feelings about a subject rather than to depict it with its natural colouring. Colour has a strong influence on the life of the people and their designs, as they tend to accept colour and recognize how each ethnic group in Ghana perceives colour. These are the components embedded in the designing of Ghanaian printed fabrics.

The prints come with very interesting Ghanaian motifs based on proverbs, adages, royal emblems, adinkra symbols among others. The choice of motif, composition, repeats etc. are carefully executed making them look very attractive when displayed. Moreover, the prints bear philosophical significance. They have names that depict or explain the beliefs and practices of the Ghanaian culture. The study sought to identify various Ghanaian printed fabrics within the Ghanaian cultural-up, examine their artistic and cultural significance vis-à-vis their impact on the general public and make an appraisal of the selected printed fabrics. It also delves into the philosophical concepts of the various designs and to ascertain the important roles they play in the Ghanaian culture.

\section{Methodology}

The study hinges on the qualitative research paradigm and employed the descriptive method. The qualitative paradigm investigates the quality of relationships, activities, situations or methods. Qualitative research also lays emphasis on holistic description i.e. on describing in detail all what goes on in a particular activity or situation. Fraenkel \& Wallen [4]. According to Ary, et al. [5] the qualitative research has the advantage of describing in narrative form (as close as possible to the form in which they are collected) and in sufficient depth and detail so that one who has not experienced the situation or event can understand and appreciate it. The designs of the fabrics were described vividly to allow easy understanding of the concepts behind them. The purposive sampling was used to select 10 printed fabrics for the study. Two textile designers and two textile retailers were also sampled for the study. The data collecting instruments used in this study were interview and observation. These instruments were used to collect data concerning artistic and cultural significance of Ghanaian printed fabrics.

Agyedu et al. [6] explained that, interview is a face-to-face meeting between a questioner and a respondent, it is often used in collecting data for descriptive studies. The interview approach helped the researchers to probe for specific meanings of responses of the study. The term observation, on the other hand, is used to indicate the object or subject of an investigation being subjected to close usually visual surveillance. It means seeing things with a purpose. Observational strategies are seen as excellent research technique to be utilized in gathering data. Affirms that, observation method is a data collection method in which a person (usually trained) observes subjects or phenomenon and records information about characteristics of the phenomenon. Careful examination of various printed fabrics 
with respect to artistic qualities such as motif arrangement, textures, colour-ways and specific uses were carried out through observation. This helped in analytical observation and appreciation of experiential analysis. These research instruments enabled the researchers to see, appreciate, photograph and helped in documentation.

\section{Discusion of Results}

To ascertain meaningful deductions from the data obtained and for a valid conclusion and relevant recommendations, descriptive analysis in the form of pictorial (Figures 1-8) of the fabrics were used. A major factor in the understanding and appreciation of art is the knowledge of its structure and compositional relationships created in the work. The structure of a work of art serves as the way the components of visual expression merge to create an organized whole. The structure is based on the organization of elements of art according to certain principles called design principles. They are generally defined as relationships created by art elements working together in a composition. They include rhythm and movement, unity, variety, emphasis, proportion, and balance etc. When the elements and principles are used together effectively, the resulting work of art can express moods, feelings, and visual messages. This therefore creates the need to be familiar with the elements and principles to appreciate the essential components and qualities of a work of art that produce desired expressive effects. It is upon this premise that the discussion of the data gathered is done [7]. The study appreciates the fabric taking into considerations the, elements and principle applied in the design composition. The descriptive analysis procedure was used based on the photographs of the fabrics identified. The analysis also focused on the artistic value, cultural significance, and the philosophical and social meanings of the fabrics as used in the Ghanaian cultural settings.

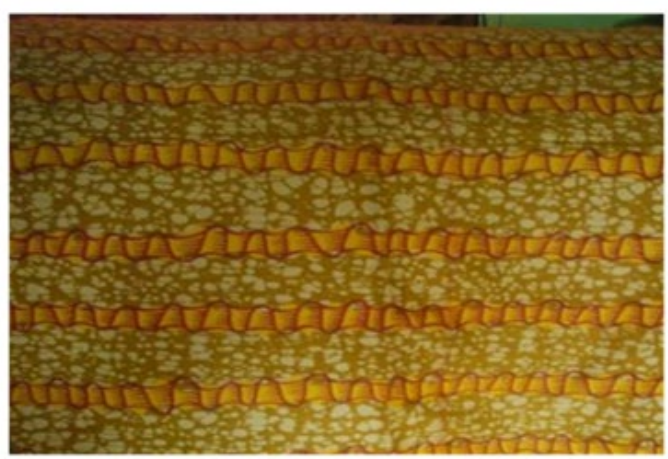

Figure 1: Awherepo.
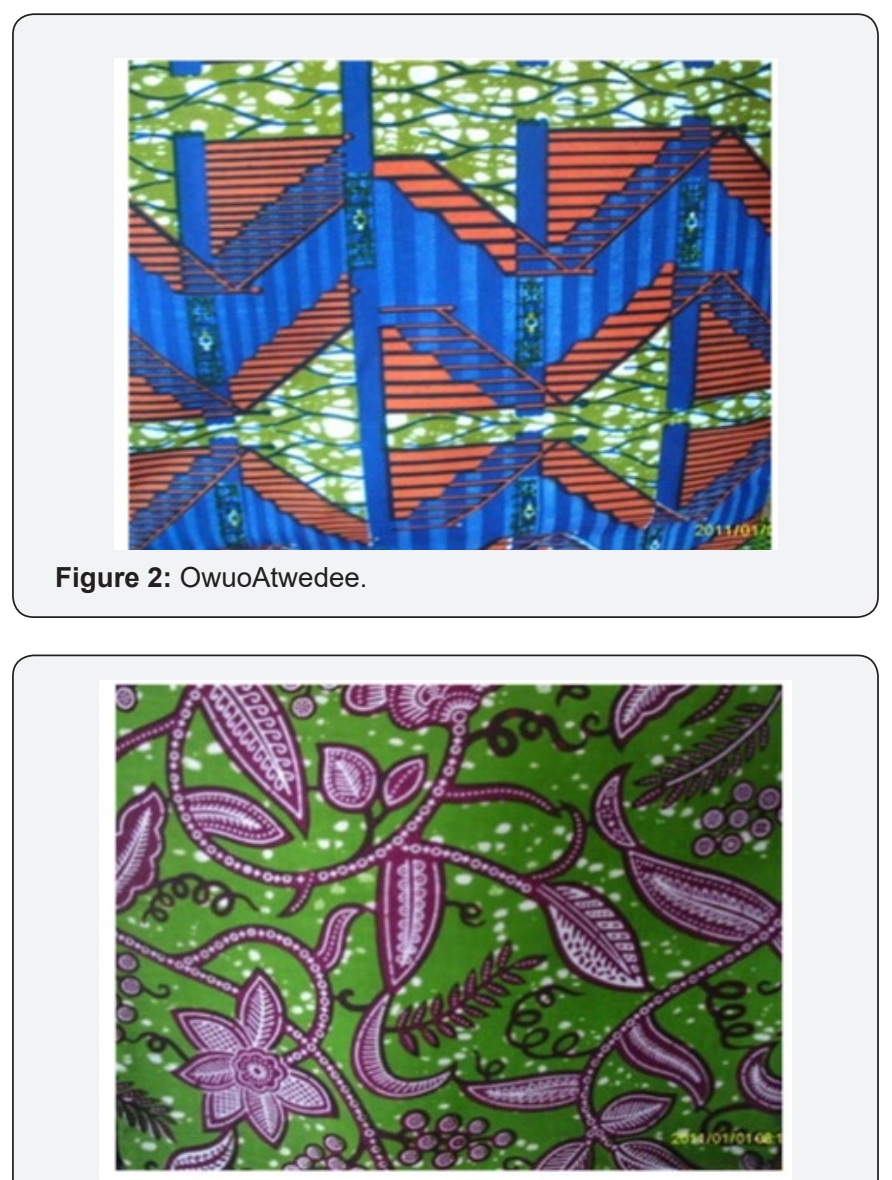

Figure 3: Ahene pa nkasa.

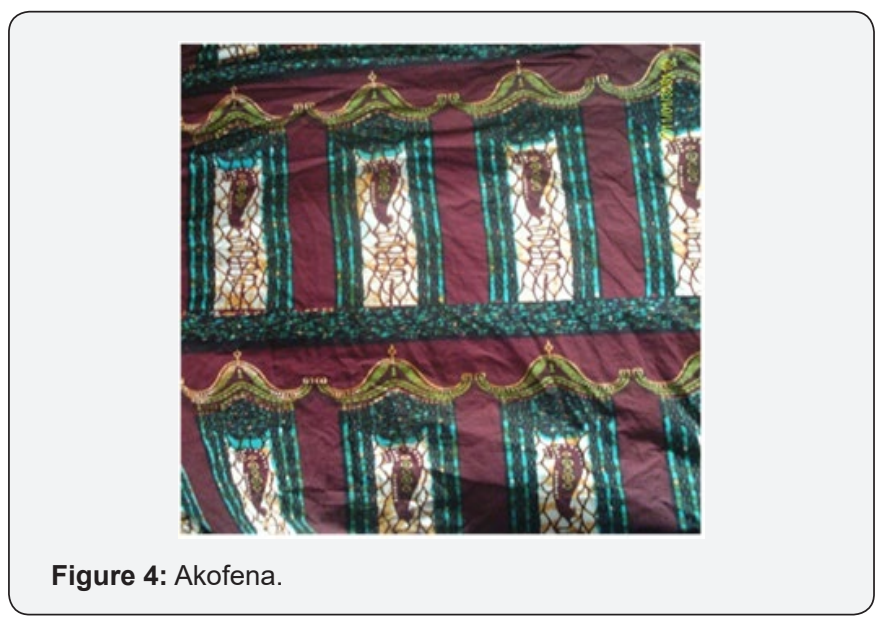




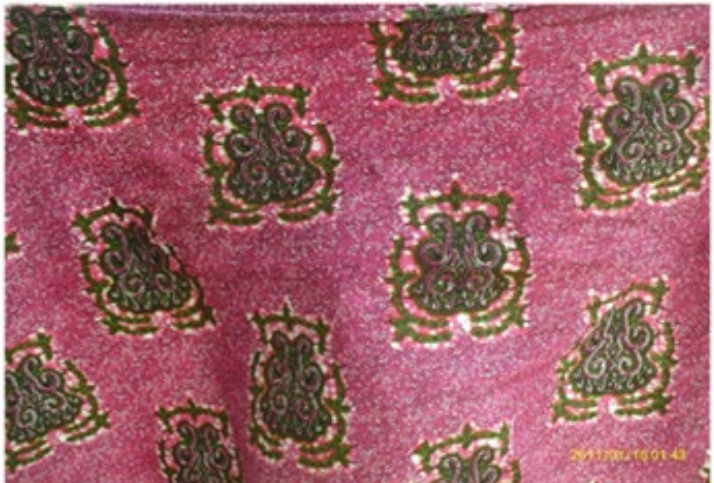

Figure 5: Akyekyedeeakyi.

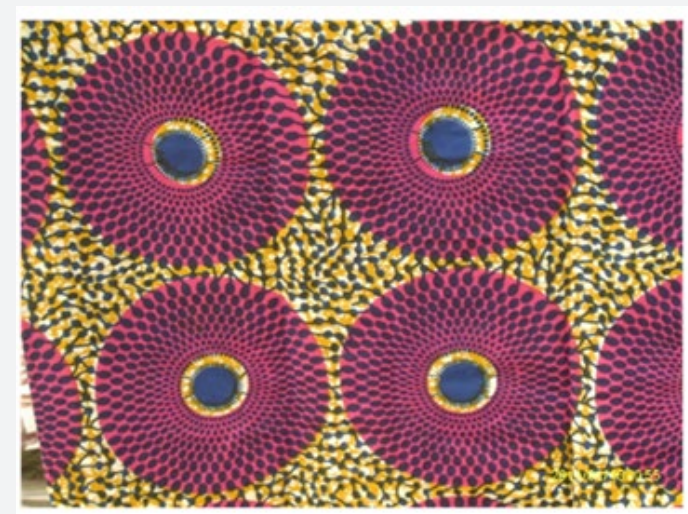

Figure 6: Nsubura.

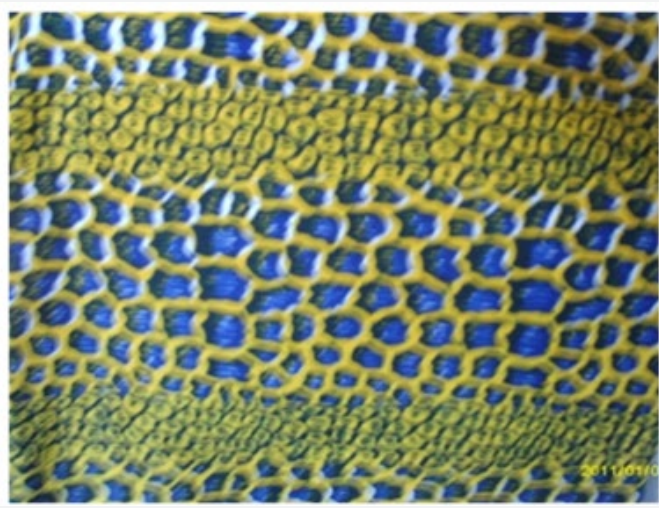

Figure 7: Efiemmosea.

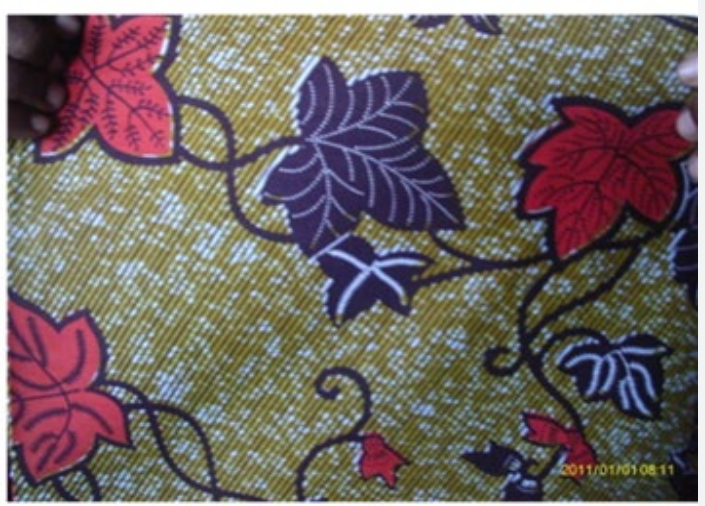

Figure 8: Felicia.

\section{Ahwerepo}

The cloth 'awherepo' is designed with series of nodes of sugar cane that has been put together either horizontally or vertically depending on the design formation. The node formation has been abstracted and characterised by wavy lines. They form a series of lines that are arranged to form the main motifs of the fabric. The background of the fabric is designed with the same nodes that has been densely arranged together to form the textures. The elements used in the design include dots, lines, shapes and colour. They have been interplayed to show variety, rhythm, contrast and harmony. The concept of harmony was used in the choice and different tints and shades of the colours to create an interesting effect on the cloth. The principle of emphasis was also used to project the nodes that were used to form the motif of the design.

"Awherepo" is an Akan word. Literally, it means sugar cane nodes. Sugar cane is a juicy cane whose sap is a source of molasses and possess commercial sugar. In its fresh state, the canes are sometimes chewed for the juice in it. It is sweet and hard. When peeling sugar cane, it is easy until it gets to the node, when it becomes difficult to peel. Significantly, it is likened to real life situation that life is like sugar cane, no matter how sweet, the juicy portion may be it becomes hard and difficult to peel when it gets to the node. Life is not all smooth, there are times when human life may face some difficulties and uncertainties. It behoves on the individual therefore to accept whatever conditions they find themselves in life believing that there are good and bad time in life. 


\section{Owuo Atwedee}

The 'Owuo Atwedie' fabric is designed with ladder, created with different types of line carefully arranged in vertical and broad horizontal patterns. The ladders were put together vertically to form zigzag lines as the main motif. The broad thick and thin lines were randomly spread on the background of the design. The arrangement of the ladders create a chain of arrow facing each other. The horizontal broad lines run through the chain of three ladders to break monotony. With a zigzag pattern of arrangement, the vertical thick lines create symmetrical balance in the design. The use of different shades of colours on the staircase creates harmonious effect. Contrast was also employed to enable the staircase stand on the background of the design. Not forgetting variety and unity that has helped to bring all the elements together as one to create an interesting design.

The elements employed in the design include lines (vertical and horizontal lines), shapes and colour. The interplay of lines creating variety of shapes such as triangles, rectangles rhombuses etc is balanced with the dots and vein-like effects created at the background. The principle of harmony employed in the creation of the background of the cloth with random curvy lines and colours that harmonizes with each other. The background textures portrayed in the wax-effect design, coupled with the wavy vein-like lines of all over patterns create an atmosphere of serenity.

"Owuo atwedee" literaly means "Owuo" - death, "atwedee" ladder. This is enshrined in an Akan proverb "owuo atwedee obaako mforo". (Death in inevitable). This symbol reflects the uncompromising nature of death. It is a necessary and an ultimate end of mankind on earth. The symbol reminds people to be modest and also live lives worthy of emulation, because death is not a respecter of persons. It also fulfils the promise of life after death and for that matter ancestor ship in particular. This is a symbol of mortality.

\section{"Ahene pa nkasa"}

The fabric "Awhene pa nkasa" is designed with floral pattern arranged using all-round technique. The floral design waves leaves come in variety of structure making it more complex in nature the floral pattern also comes with a grape-like fruit of beautiful.

The elements employed in the design include, floral design, lines (curvy, diagonal and spiral lines), dots, shape and colour. The design has the wax printing method for the background of the design and the block printing technique for the floral pattern. The elements in the design are harmonious as a result of the use of cool colour and the white spots on the background and white lines, shapes and dots used on the floral design. Contrast was achieved in the use of white on the violet background of the floral design. Variety and unity can be seen in the use of varied elements coming together as one to create a pleasant atmosphere. "Ahwene pa nkasa" means "precious or quality beads make no noise". This adage implies empty barrels make the most noise or a good person needs not blow his own horns.

\section{"Akofena"}

The items in the design include the sword, wavy lines, curvy likes, doodle-like pattern and geometric boarder design. The wax printing technique was used for the treatment of the background of the block print. The designer made good use of the principle of contrast to enable the boarded background with the main motif stand on it. The sword with three geometric designs on it is caged in rectangular shape, forming the main motif of the design. The principle of harmony was also employed to create harmonious effect in the design. Unity and variety were also used to bring the various elements together as one with a common ideology.

Akofena literally means 'ako' - war, 'afena' - sword. (War sword or ceremonial sword). This symbol signifies the power, strength and authority vested in leader. It also symbolises the gallantry and loyalty of warriors who protect a particular chieftain. It is also very significant for swearing the oath of allegiance. The symbol advises people to honour and show loyalty to their leaders. It also encourages statesmanship and gallantry. This is a symbol of authority and gallantry. Ceremonial sword also signifies the social standing of clan, group or state in terms of strength, wealth and attitudes. The state sword repeated several times in the fabric, is a design usually worn by elderly wealthy and powerful people in the society.

\section{Akyekyedee akyi}

"Akyekyedee akyii" is a design with the shell of the tortoise with intricate designs on it and fence-like pattern around the shell to form the main motif. The background of the cloth is designed with shaded colour randomly spread in (all-round pattern)

The elements used in the design include dots, fence-like design and colour creating an intricate design. The half drop technique was used in the arrangement of the main motif of the fabric. A conflicting was created in the created in the choice of colours that were used for the motif and its background. The way the dots have been treated using all-round pattern, creates a harmonious effect on the background. Symmetric balance was also used in the designing of the main motif of the fabric and unity in the organization of the intricate designs on the tortoise shell to enhance the pattern.

'Akyekyedee akyii" meaning' the shell of a tortoise'. The shell of the tortoise is very hard and it protects its skin. In the Ghanaian community, clans use the tortoise as their totem. The tortoise moves slowly due to its nature. For this reason it is independent and self-sufficient. The tortoise hides itself under 
the hard shell to prevent it from being hurt. The back has been intricately designed to show the beauty of the back.

\section{Nsubura}

The main motif of the fabric is designed with blue oval and a moon-like shaded blue to form a complete circle, a purple circular shape. The purple circular shape is surrounded by multiple oval shapes arranged in a circular form from the smallest to the biggest on a yellowish green background. The motif is arranged using the full-drop pattern repeat on a purple background with shaded blue spots connected with each other with the aid of short lines. The design gives the impression of radial balance.

The textile designer made good use of the following elements: colour, shape and line. He/she employed the fulldrop pattern repeat in the arrangement of the main motif of the design and all-over pattern repeat was also used in the treatment of the background. The choice of colour used helped to achieve contrast and harmony in the use of shaded blue on yellow and shaded blue on violet respectively. Balance was achieved in the arrangement of the motif. Not forgetting variety and unity that were used together to bring the various elements together as one fabric.

"Nsubura" or "nubu" (Ga) literally means deep well of water. Water extracted from deep down the earth or underground is clean or has been purified by nature. Before one gets this clean or purified water it needs hard work, since you have to dig deep to get the water. Among the Ewes during their "agbadza" dance, they have this lyrics "Gbedze vor medeo te kpo ee amewo te ko dom abodzi". They use the name of the cloth to sing. The song simply means 'those who have never use the cloth now feel proud of using it the first time'. They dance proudly by turning the head towards the shoulder. This means they are happy and feels proud to use the cloth.

\section{Efie mmosea}

The fabric is designed with an irregular stone-like shape randomly arranged in a concrete and abstract/inverse form to create strips. The stone-like shapes have spaces in between them. The elements used are stone-like shapes, colour and lines. The all-over repeat pattern was used to arrange the motif. Contrast was used to create conflict between the background and the foreground colours. The principle of unity and variety were also used to put the stone-like shapes together to create harmonious effect on the design. 'Efie mmosea' means house-hold pebbles. It implies that only close relations can hurt you badly or without your close relatives' involvement, no one can hurt you. Among the Ewes and Gas ethnic groups, it means the poor man does not chew stones. No one chew stones in the Ghanaian community. We are one another's keeper, therefore when your neighbour is in trouble, the members in the society deem it a duty to help. Members of the society help one another for unity.

\section{Felicia}

Diagonal lines in brown moving from right to left on a tinted brown background with white stain-like spots. The fabric comes with a print of pink leaves with shaded indigo outline and veins; and indigo blue leaves with white veins. The leaves are connected with curvy lines to form the main pattern of the cloth arranged all-round.

The elements employed in the designing of the fabric include lines (diagonal and curvy lines), colour and shape (leaves). Wax print technique was used for the treatment of the background. The tinted brown, brown diagonal lines and stain-line white spots creates harmonious effect as background of the cloth. Unity was also used to bring the elements together while contrast was employed in the choice of colours for the background and the motifs and outline colours.

Felicia is an English female name. Oral tradition has it that a man travelled and on his return bought the floral designed fabric for the beloved wife Felicia. Due to the graceful nature of the floral design in the fabric, the man told the wife that the cloth is called Felicia hence the name.

\section{Conclusion}

The research was to identify and select Ghanaian printed cloths designs, look at their cultural significance and the aesthetic value inherent in the designs centred on Ghanaian identity. The following conclusions are made based on the study that, fabrics have names that have direct correlation to the significance of the fabric. These names given to the fabrics are provided based on some cultural and traditional beliefs. Indeed, the aesthetic values and cultural significance are based on some cultural beliefs and that these beliefs are either symbolically presented in the design of the fabric. The designs of these fabrics are used across-culture in Ghana. The names given are based on the crosscultural behaviour of the people. The fact that some of the names of the Ghanaian printed fabrics are in the local languages is a good thing as it enhances a sense of belonging and appreciation of the Ghanaian spoken languages. The fabrics have their own aesthetics and cultural significance that are appreciated by the entire cross-section of the Ghanaian populace. The use of these fabrics is done, based on the aesthetic and functional purposes of the individuals concerned. The complex and intricate nature of these designs coupled with the variety of colour-ways available portray the love for colours within the Ghanaian cultural set-up. Various shapes and sizes are used with different colours that are representative of the particular design being portrayed in the design. The design makes good use of elements like line, shape, colour and texture (visual texture). The principle of movement was employed in the way the diagonal lines on the yellow squares have being used. The study recommendations that, conscious efforts be made by the various centres of national cultures to 
have special collections of these fabrics as means of beefing up the stock of knowledge that exists in the meanings of the fabrics designs.

\section{References}

1. Tucker, Barbara M (1981) The merchant, the manufacturer, and the factory manager: The case of Samuel Slater. Business History Review 55(3): 297-313.

2. Hofstede E (1997) Definition of culture. Annual Review of Anthropology 12(5): 12-30.

3. Amenuke SK, Dogbe BK, Asare FK, Ayiku RK, Baffoe A (1991) General knowledge in Art for senior secondary schools, Evans Brothers Ltd., London, UK.
4. Frankael JR, Wallen NE (2002) How to design and evaluate research in education. Mcgraw-Hill, New York, USA.

5. Ary D, Jacobs LC, Razaviel A (1990) Introduction to research in education $\left(4^{\text {th }}\right.$ edn). Holt, Rinehart \& Winston, Inc, Orlando, US.

6. Agyedu GO, Donkor F, Obeng S (2011) Teaching yourself research methods, University of Education, Winnba. Kumasi Campus, Ghana.

7. Bates RJ (2006) Aesthetic dimension of educational administration and leadership, Routledge, London, UK.

DOI: 10.19080/CTFTTE.2018.02.555594 